\title{
COMMENT
}

Check for updates

\section{T cell-oriented strategies for controlling the COVID-19 pandemic}

Ji Yun Noh ${ }^{1,2}$, Hye Won Jeong ${ }^{1,3}$, Jerome H. Kim (10 ${ }^{4}$ and Eui-Cheol Shin (10) ${ }^{1,5 \bowtie}$

COVID-19 vaccination programmes are ongoing worldwide. Neutralizing antibodies are thought to be key for host protection against COVID-19; however, strategies that focus only on neutralizing antibodies may not be sufficient to cope with the pandemic in the longer term owing to the decay of antibody titres and the emergence of antibody-escape variants of SARS-CoV-2. Here, we describe the protective roles of T cells in COVID-19 and the conservation of T cell epitopes in SARS-CoV-2 variants of concern, and discuss the potential contribution of T cell-oriented strategies to controlling the COVID-19 pandemic.

'Laboratory of Immunology and Infectious Diseases, Graduate School of Medical Science and Engineering, Korea Advanced Institute of Science and Technology (KAIST), Daejeon, Republic of Korea.

${ }^{2}$ Division of Infectious Diseases, Department of Internal Medicine, Korea University College of Medicine, Seoul, Republic of Korea.

${ }^{3}$ Division of Infectious Diseases, Department of Internal Medicine, Chungbuk National University College of Medicine, Cheongju, Republic of Korea.

4International Vaccine Institute, Seoul, Republic of Korea.

${ }^{5}$ The Center for Epidemic Preparedness, KAIST, Daejeon, Republic of Korea

凶e-mail:ecshin@kaist.ac.kr https://doi.org/10.1038/ s41577-021-00625-9
COVID-19 vaccines induce both virus-specific antibodies and $\mathrm{T}$ cell responses, but it is the neutralizing antibodies (nAbs) that interfere with the entry of SARS-CoV-2 into host cells that are considered to be key for host protection. However, the level of nAbs declines with time after SARS-CoV-2 infection or vaccination ${ }^{1}$. Moreover, recently emerging SARS-CoV-2 variants can escape $n$ Abs elicited by natural infection or vaccination ${ }^{2}$.

During a typical virus infection, $\mathrm{CD} 4^{+}$and $\mathrm{CD} 8^{+}$ $\mathrm{T}$ cells contribute to controlling the virus by producing effector cytokines, such as IFN $\gamma$ and TNF, and by exerting cytotoxic activity against virus-infected cells. Indeed, early and robust SARS-CoV-2-specific $\mathrm{T}$ cell responses have been associated with limiting the severity of COVID-19 (REF. ${ }^{3}$ ). In a study examining SARS-CoV-2-specific immune responses in patients with acute COVID-19, there was a strong inverse correlation between the frequency of SARS-CoV-2-specific IFN $\gamma$-producing $\mathrm{CD}^{+} \mathrm{T}$ cells and disease severity ${ }^{3}$. In addition, $\mathrm{CD}^{+} \mathrm{T}$ cells specific for conserved epitopes across common cold coronaviruses (CCCoVs) are enriched in patients with mild COVID-19 (REF. $\left.{ }^{4}\right)$. Given that individuals not exposed to SARS-CoV-2 have cross-reactive $\mathrm{T}$ cells primed by previous $\mathrm{CCCoV}$ infection, this suggests that pre-existing $\mathrm{T}$ cell immunity could ameliorate progression to severe COVID-19.

Intriguingly, host protection mediated by $\mathrm{T}$ cells has been observed in patients with COVID-19 who have insufficient humoral immune responses. Among patients with a haematological malignancy who became infected with SARS-CoV-2, anti-CD20 therapy was not associated with increased fatality, disease severity or viral load, despite low titres of SARS-CoV-2-specific IgG ${ }^{5}$. In these patients, including those receiving anti-CD20 therapy, SARS-CoV-2-specific $\mathrm{T}$ cell responses were detected, and higher levels of $\mathrm{CD}^{+} \mathrm{T}$ cell-mediated immunity were associated with improved patient survival.
Similarly, the protective roles of T cell-mediated immunity against SARS-CoV-2 have been demonstrated in animal models. Mice immunized with a vaccine expressing SARS-CoV-2 T cell epitopes exhibited decreased viral titres and reduced lung pathology when challenged with SARS-CoV-2, despite the absence of nAbs, which indicates that SARS-CoV-2-specific T cell vaccination confers partial protection from severe disease $^{6}$. In a macaque model, $\mathrm{CD} 8^{+} \mathrm{T}$ cell depletion in convalescent animals was shown to partially abrogate host protection against SARS-CoV-2 re-challenge ${ }^{7}$. Together, these results in human patients and animal models indicate that $\mathrm{T}$ cells have a host protective role during COVID-19, particularly when the humoral immune response is insufficient.

SARS-CoV-2 nAbs elicited by infection or vaccination might become insufficient for host protection owing to declining titres over time and/or the emergence of viral escape variants. Although the kinetics of $n A b$ titres vary among COVID-19 convalescent individuals, more than half of these individuals have waning levels of $n A b s$ after 6 months ${ }^{1}$. Moreover, SARS-CoV-2 variants of concern (VOCs) that have emerged have resulted in considerably reduced activity of nAbs induced by previous infection or vaccination. Sera from convalescent individuals and vaccine recipients have significantly weakened neutralizing activities against the Beta (B.1.351) and Delta (B.1.617.2) variants of SARS-CoV-2 (REF. ${ }^{2}$ ). The emergence and rapid rise to global predominance of the Delta variant, together with the emergence of the Lambda variant (C.37), remind us that VOCs are likely to continue to evolve and challenge existing vaccines that depend primarily on humoral immune responses.

Compared with nAbs, SARS-CoV-2-specific memory $T$ cells are maintained for a relatively long time (TABLE 1). A recent study showed that SARS-CoV-2-specific memory $\mathrm{CD}^{+}$and $\mathrm{CD}^{+} \mathrm{T}$ cell responses are sustained 
Table 1 | Neutralizing antibodies and memory T cells elicited by different COVID-19 vaccine strategies

\begin{tabular}{|c|c|c|c|c|}
\hline $\begin{array}{l}\text { Type of vaccine } \\
\text { antigen(s) }\end{array}$ & $\begin{array}{l}\text { Form of protective } \\
\text { immunity }\end{array}$ & Clinical effects & Longevity & Escape by virus variants \\
\hline Spike (S) protein or gene & $\begin{array}{l}\text { Neutralizing antibodies } \\
\text { that block viral entry } \\
\text { into host cells }\end{array}$ & $\begin{array}{l}\text { Protection from } \\
\text { infection and from } \\
\text { severe disease }\end{array}$ & $\begin{array}{l}\text { Relatively } \\
\text { short }\end{array}$ & $\begin{array}{l}\text { Easily escaped by mutations } \\
\text { in antibody-binding sites }\end{array}$ \\
\hline $\begin{array}{l}\text { Spike (S) protein or } \\
\text { gene; other structural } \\
\text { and non-structural viral } \\
\text { proteins or genes, or T cell } \\
\text { epitopes }\end{array}$ & $\begin{array}{l}\mathrm{CD} 4^{+} \text {and } \mathrm{CD} 8^{+} T \text { cells } \\
\text { that produce cytokines } \\
\text { and kill infected cells }\end{array}$ & $\begin{array}{l}\text { Protection from } \\
\text { severe disease }\end{array}$ & $\begin{array}{l}\text { Longer } \\
\text { lasting }\end{array}$ & $\begin{array}{l}\text { Much harder to escape } \\
\text { because HLA-restricted } \\
\text { T cell epitopes vary between } \\
\text { individuals and are distributed } \\
\text { widely across a protein }\end{array}$ \\
\hline
\end{tabular}

in COVID-19 convalescent individuals for 10 months regardless of disease severity ${ }^{8}$. The persistence of memory $\mathrm{T}$ cell responses to SARS-CoV for 17 years has been demonstrated ${ }^{9}$. Moreover, there is increasing evidence that SARS-CoV-2 VOCs rarely escape memory T cell responses elicited by SARS-CoV-2 infection or vaccination. One study showed that SARS-CoV-2-specific $\mathrm{T}$ cell reactivity in COVID-19 convalescent individuals and vaccine recipients is reduced by only $10-22 \%$ by virus variants, including the Alpha (B.1.1.7), Beta, Gamma (P.1) and Epsilon (B.1.429) variants ${ }^{10}$. By analysing amino acid sequences, it was shown that $93 \%$ and $97 \%$ of the $\mathrm{CD}^{+}$and $\mathrm{CD}^{+} \mathrm{T}$ cell epitopes, respectively, were conserved in these variants. In principle, it is more difficult for a virus to evade $\mathrm{T}$ cell responses than $\mathrm{nAbs}$ because multiple $\mathrm{T}$ cell epitopes are distributed across both structural and non-structural viral proteins, including internal virus components, whereas nAbs tend to target a restricted protein domain exposed on the virus surface - such as the spike (S) protein of SARS-CoV-2, which is targeted by the currently approved viral vector and mRNA vaccines for COVID-19.

Currently, other vaccines with multiple targets, not limited to the SARS-CoV-2 S protein, are under development that should induce broad $\mathrm{T}$ cell responses. For example, UB-612, a protein-based vaccine incorporating multiple $\mathrm{CD}^{+}$and $\mathrm{CD}^{+} \mathrm{T}$ cell epitopes selected from the matrix $(\mathrm{M}), \mathrm{S} 2$ and nucleocapsid $(\mathrm{N})$ proteins of SARS-CoV-2, is in a phase II/III clinical trial (NCT04683224). Using a DNA platform, GX-19N expressing S and N proteins (NCT04715997) and GLS-5310 expressing S and ORF3a proteins (NCT04673149) are in phase I/II trials. There are also adenovirus vector vaccines expressing $\mathrm{S}$ and $\mathrm{N}$ proteins in development, including hAd5-S-Fusion + N-ETSD (NCT04843722) and VXA-CoV2-1 (NCT04563702). Chimpanzee adenovirus and self-amplifying mRNA vector vaccines expressing $\mathrm{S}$ protein and additional $\mathrm{T}$ cell epitopes (NCT04776317) and a synthetic modified vaccinia Ankara viral vector vaccine encoding $\mathrm{S}$ and $\mathrm{N}$ proteins (NCT04977024) are under investigation. Peptide vaccines using $\mathrm{CD}^{+}$or $\mathrm{CD} 8^{+} \mathrm{T}$ cell epitopes are being developed (NCT04885361), particularly for adults with congenital or acquired humoral immune deficiency (NCT04954469).
It is not likely to be possible to achieve herd immunity against the current COVID-19 pandemic owing to the decay of $\mathrm{nAb}$ titres and the continuing emergence of escape variants. A practical goal may therefore be to reduce damage to public health by making COVID-19 a controllable disease, similar to influenza or the common cold, although the elderly and immunocompromised patients would still be at risk of severe disease. We therefore need to continue work on such vaccines that induce durable and broad protective T cell-mediated immunity against COVID-19.

In the meantime, we should continue current vaccination programmes using the approved COVID-19 vaccines, which are still partially effective against SARS-CoV-2 VOCs, particularly in the prevention of severe or lethal COVID-19. Furthermore, SARS-CoV-2 breakthrough infections seem to be characterized by mild illness, low viral load and reduced (but not negligible) transmission risk. We believe that $\mathrm{T}$ cells could provide a solid defence against COVID-19 secondary to the activity of nAbs. Thus, sophisticated T cell-oriented vaccine strategies should be considered to control the COVID-19 pandemic in the longer term.

1. Chia, W. N. et al. Dynamics of SARS-CoV-2 neutralising antibody responses and duration of immunity: a longitudinal study. Lancet Microbe 2, e240-e249 (2021).

2. Planas, D. et al. Reduced sensitivity of SARS-CoV-2 variant Delta to antibody neutralization. Nature 596, 276-280 (2021).

3. Rydyznski Moderbacher, C. et al. Antigen-specific adaptive immunity to SARS-CoV-2 in acute COVID-19 and associations with age and disease severity. Cell 183, 996-1012 (2020).

4. Mallajosyula, V. et al. CD8 ${ }^{+}$T cells specific for conserved coronavirus epitopes correlate with milder disease in COVID-19 patients. Sci. Immunol. 6, eabg5669 (2021).

5. Bange, E. M. et al. CD8 $8^{+} \mathrm{T}$ cells contribute to survival in patients with COVID-19 and hematologic cancer. Nat. Med. 27, 1280-1289 (2021).

6. Zhuang, Z. et al. Mapping and role of T cell response in SARS-CoV-2infected mice. J. Exp. Med. 218, e20202187 (2021).

7. McMahan, K. et al. Correlates of protection against SARS-CoV-2 in rhesus macaques. Nature 590, 630-634 (2021).

8. Jung, J. H. et al. SARS-CoV-2-specific T cell memory is sustained in COVID-19 convalescent patients for 10 months with successful development of stem cell-like memory T cells. Nat. Commun. 12, 4043 (2021).

9. Le Bert, N. et al. SARS-CoV-2-specific T cell immunity in cases of COVID-19 and SARS, and uninfected controls. Nature 584, 457-462 (2020).

10. Tarke, A. et al. Impact of SARS-CoV-2 variants on the total $\mathrm{CD}^{+}$and $\mathrm{CD}^{+} \mathrm{T}$ cell reactivity in infected or vaccinated individuals. Cell Rep. Med. 2, 100355 (2021).

\section{Acknowledgements}

This work was funded by Samsung Science and Technology Foundation under project number SSTF- BA1402-51. 